\title{
Potential physio-pathological effects of branched fatty acid esters of hydroxy fatty acids
}

\author{
Melha Benlebna a ${ }^{\text {, }}$ Laurence Balas ${ }^{\mathrm{b}}$, Sylvie Gaillet ${ }^{\mathrm{a}}$, Thierry Durand ${ }^{\mathrm{b}}$, Charles Coudray ${ }^{\mathrm{a}}$, \\ François Casas ${ }^{a}$, Christine Feillet-Coudray ${ }^{\text {a, * }}$
}

a DMEM, INRAE, Univ Montpellier, Montpellier, France

b IBMM, Univ Montpellier, CNRS, ENSCM, Montpellier, France

\begin{abstract}
A B S T R A C T
Branched Fatty Acid Esters of Hydroxy Fatty Acids (FAHFAs) are a new endogenous lipid class with recently uncovered interesting biological effects and which have been detected in food of plant and animal origins. Some FAHFAs can improve glucose tolerance and insulin sensitivity, stimulate insulin secretion, and exert anti-inflammatory effects. Other beneficial health effects have also been suggested, in particular against some cancers. FAHFAs could therefore be a potential therapeutic target for the treatment of numerous metabolic disorders such as type II diabetes, hepatic steatosis, cardiovascular diseases and various cancers. Their recent discovery has generated a great interest in the field of human health. This short review aims at bringing together the information available to date in the literature concerning their chemical synthesis, biosynthesis and degradation pathways as well as their potential physio-pathological beneficial effects.
\end{abstract}

\author{
Keywords: \\ FAHFAs \\ Bioactive lipids \\ Metabolism \\ Diabetes \\ Obesity \\ Inflammation \\ Cancer
}

\section{Contents}

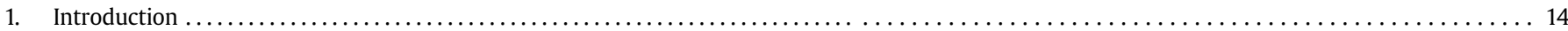

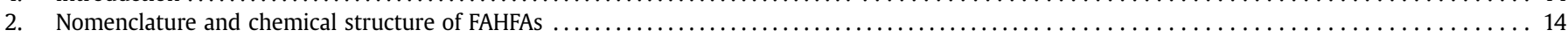

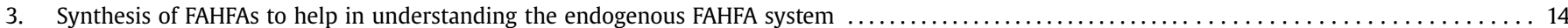

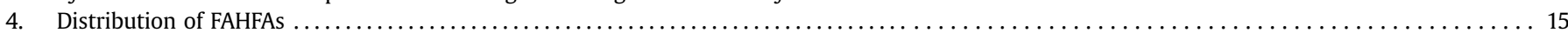

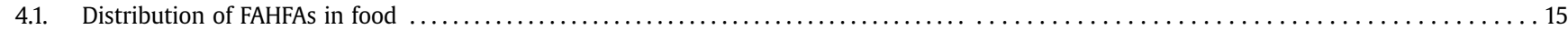

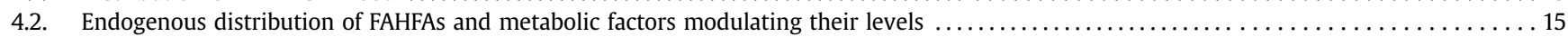

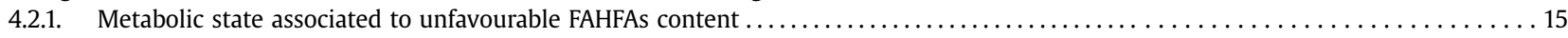

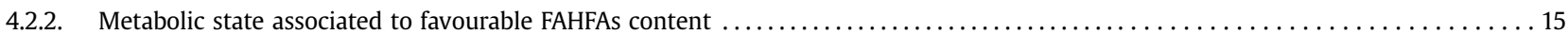

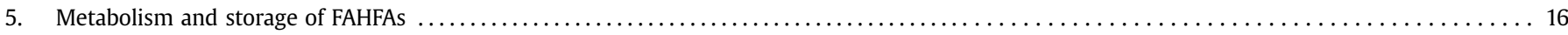

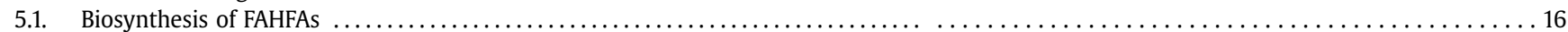

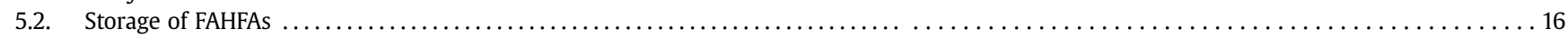

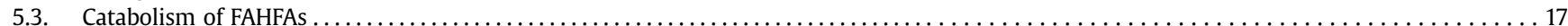

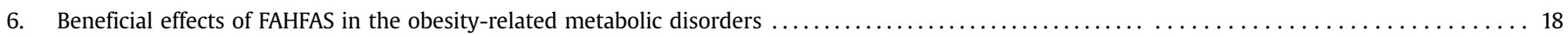

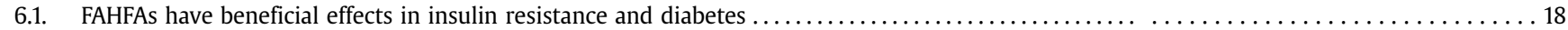

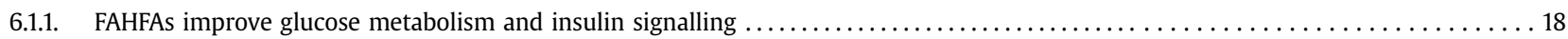

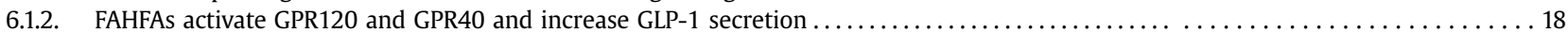

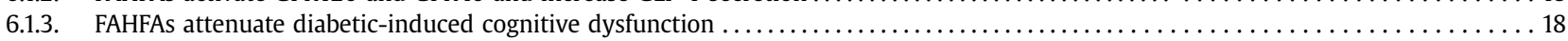

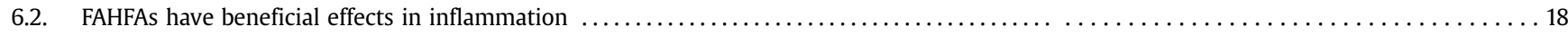

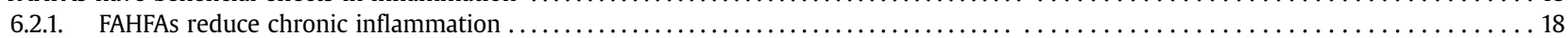

\footnotetext{
* Corresponding author. INRAE, UMR 866, 34060, Montpellier, France.

E-mail address: christine.coudray@inrae.fr (C. Feillet-Coudray).
} 


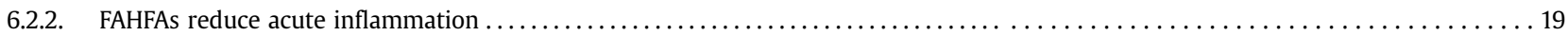

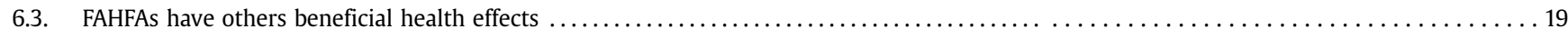

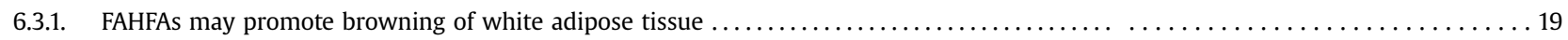

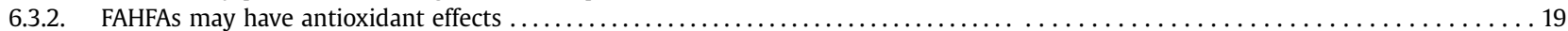

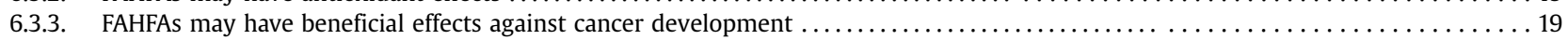

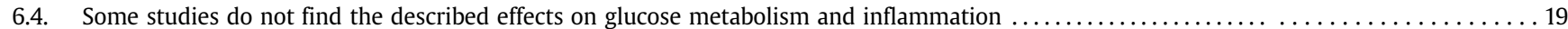

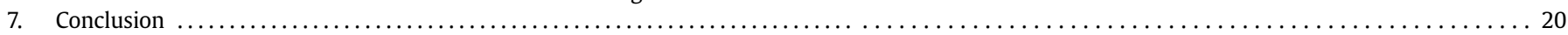

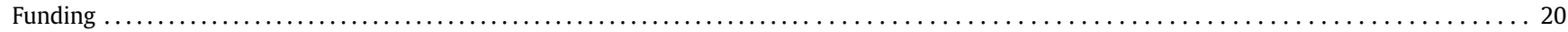

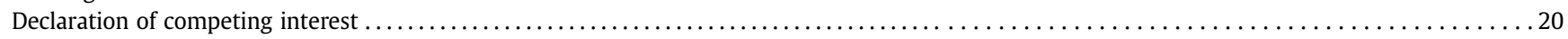

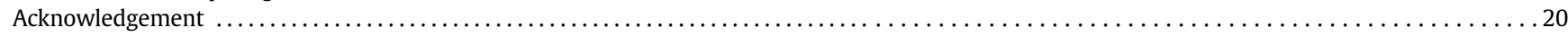

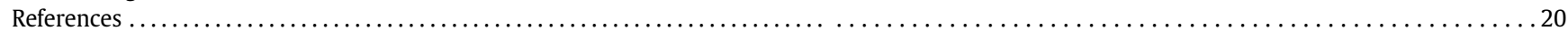

\section{Introduction}

Over the past 50 years, obesity and sedentary lifestyles have increased dramatically in industrialized countries and more recently in emerging countries, with impacts on health and wellbeing of populations. Obesity is a major public health issue as several studies have shown that being overweight and obese are the main causes of the development of metabolic disorders (such as type II diabetes, hepatic steatosis, cardiovascular diseases and various cancers), which can lead to significant morbidity and mortality [1]. Branched Fatty Acid Esters of Hydroxy Fatty Acids (FAHFAs) are a family of endogenous lipids with appealing biological effects, recently uncovered by Pr Kahn's team [2] and which have been detected in food of plant and animal origin. Some members of this class of lipids have anti-diabetic and antiinflammatory activity $[2,3]$. These lipids have therefore generated a great interest in the field of human health. Information about their biosynthesis, degradation pathways and their potential biological effects remains partial. This review aims at collecting the information available to date in the literature concerning health beneficial effects of FAHFAs.

\section{Nomenclature and chemical structure of FAHFAs}

The nomenclature of FAHFAs is based on their chemical structure, using well-known acronyms of the non-hydroxylated fatty acid (FA) and the hydroxylated fatty acid (HFA). Some examples are depicted in Fig. 1.

Naturally occurring FA and HFA are numerous. Thus, multiple combinations of FA and HFA are possible, generating hundreds of FAHFAs [2,4]. To date, almost 50 families of FAHFAs have been identified including seven FA: palmitic acid (PA), palmitoleic acid (PO), stearic acid (or SA), oleic acid (OA), linoleic acid (LA), arachidonic acid (AA), docosahexaenoic acid (DHA) and their corresponding HFA (HPA, HPOA, HSA, HOA, HLA, HAA and HDHA) [2,4], the esters of palmitic acid and hydroxy stearic acid (PAHSA) being the most studied. In each family of FAHFAs, several positional isomers are possible and in fact more than 300 regioisomers have been currently identified [5]. For example, 5-PAHSA and 9-PAHSA are two regioisomers that have the ester bond at 5- or 9- position respectively (numbering from the carboxylate).

\section{Synthesis of FAHFAs to help in understanding the endogenous FAHFA system}

The discovery of saturated branched fatty acid esters with health benefits shakes up conventional thinking that usually associated saturated fatty acids to "bad fat", mainly useful for energy storage. As a result, this renewed interest in saturated fatty acids (FA) and hydroxyl fatty acids, (HFA), combined with the limited availability of FAHFAs from natural and endogenous sources have sparked a great interest in their total chemical synthesis. The first multistep syntheses were performed for preliminary biological evaluation and lipidomic analysis. In this context, racemic FAHFAs were provided as standards for calibration curves, co-injection, MS-MS fragmentation and retention time comparison for their detection and identification in many natural sources and biological samples/ tissues (see section IV).

A review covering the different chemical strategies to access saturated FAHFAs has been recently published by Balas et al. [6]. Since then, beside the challenge of a regiocontrolled synthesis to introduce the hydroxyl group at a well-defined position in the HFA chains, organic chemists aimed at developing shorter and more concise synthesis strategies, avoiding the protecting groups [7] or using a selective methyl ester saponification in the presence of the FAHFA ester linkage [8-10]. Structure-activity relationship was explored with libraries of short chain FAHFAs, namely some truncated PAHSA regioisomers [9] or regioisomers of lauric acid [7,11].

Beside in vitro biological experiments, synthetic FAHFAs also serve for in vivo investigation of their biological effects. For that purpose, the multistep syntheses have been scaled up for use in several months diet supplementation [12-15] or gavage in mice $[16,17]$ and have been applied to the preparation of deuterated or

1) 9-PAHPA

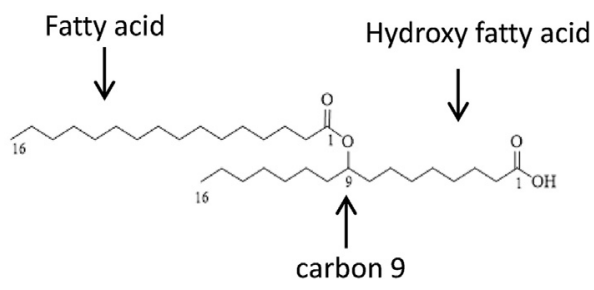

2) 9-OAHPA

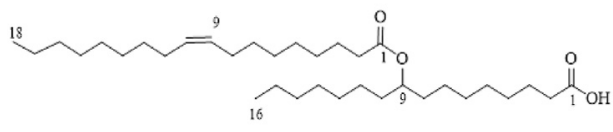

3) 5-PAHPA

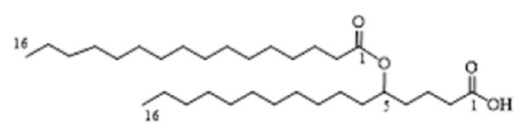

Fig. 1. Chemical structure of some FAHFAs.

1) 9-PAHPA: palmitic acid (PA, C16:0) is esterified at the 9th carbon of hydroxy palmitic acid, 2) 9-OAHPA: oleic acid (OA, C18:1) is esterified at the 9th carbon of hydroxy palmitic acid, 3) 5-PAHPA: palmitic acid is esterified at the 5th carbon of hydroxy palmitic acid (SA, C16:0). 
${ }^{13} \mathrm{C}$ labeled FAHFAs.

The configuration of the chiral center was shown [18] to be of great importance for the rate of their biosynthesis and ester linkage hydrolysis (see section V). Thus, for the last three years, organic chemists focused their efforts on stereocontrolled multistep strategies, providing several enantio-pure or enantio-enriched FAHFAs, using (R)- and (S)-chiral pools [epichlorohydrin epimers [18,19]; (S)-glycidol [20]; (R)-glyceraldehyde [21]; D-glucose derivatives [22-24] or asymmetric enantioselective reactions (asymmetric Jorgensen epoxidation [25,26]; asymmetric allylations [27,28]; lipase-catalyzed transesterification [29]; asymmetric hydrogenation [30], respectively. Both (R)- and (S)- enantiomers were detected in vivo [18,31].

Structurally, branched FAHFAs are not restricted to the class of saturated or monounsaturated lipids. Recently chemists have shown FAHFAs containing a polyunsaturated FA chain, a polyunsaturated HFA chain or both. Thus, challenging regio- and stereoselective total synthesis of several racemic or optically active polyunsaturated FAHFAs have been performed. Hansen's group has recently prepared [20] 13(R)- and 13(S)-DHAHLA enantiomers, showing higher anti-inflammatory properties than 9-PAHSA [4,32]. The same year, Siegel's team synthesized [19] three antiinflammatory [33] LAHLA regioisomers i.e. 15(R)-LAHLA observed as a digalactosyldiacylglycerol conjugate in oat $[34,35]$ and the racemic 9- and 13-LAHLAs.

More recently, synthetic FAHFAs were also used as starting material for the preparation of FAHFA-containing triacylglycerols (TAGs). The discovery of these TAG-estolides [36,37] is an important breakthrough in the understanding of the role of FAHFAs in producing remodeling of FA profiles (see section $\mathrm{V}$ ). Since only tiny amounts of such FAHFAs were needed for LC-MS/MS detection, chemists carried out short regioselective syntheses via direct (modified) Steglich esterification of (di)acylglycerols, without paying too much attention to the efficiency of the chemical strategies [17,37]. To date, while the strategies control the number of FAHFAs and its/their position in the glycerol backbone, the chirality of both the FAHFAs and glygerol stereogenic centers has not yet been taken into consideration.

Over the past six years, considerable progress in the understanding of FAHFA system has been achieved, thanks to the many saturated and polyunsaturated synthetic FAHFA compounds designed as tools for lipidomic analysis, mechanism studies, physio(patho)logical relevance and biological roles (see section VI). More details about their total synthesis (schemes, comparison of the chemical strategies, ...) have recently been reviewed [17].

\section{Distribution of FAHFAs}

Using a supplementation by gavage in mice with a nonendogenous HFA, namely 9-hydroxy heptadecanoic acid (9-HHA, usually not present in mammalian tissues), Yore et al. [2] showed that FAHFAs can be synthesized in vivo. However, FAHFAs can also be obtained exogenously from food.

\subsection{Distribution of FAHFAs in food}

A tremendous effort has been devoted to the development of analytical methods for the detection and quantitation of FAHFAs during the last five years [38]. Numerous FAHFA families have been detected in food of plant origin (fruits, vegetables and cereals) $[39,40]$ and of animal origin (egg, chicken, beef, caribou, moose) $[2,41]$. Abundance of FAHFAs varies according to the type of food considered (see Table 1). It should be noticed that their abundance also varies according to their chemical structure. Yore et al. identified 5 regioisomers of PAHSA in food of animal origin [2]. Liberati-
Čizmek et al. [39] detected 16 different FAHFAs in food of plant origin and Zhu et al. [40] detected 49 potential FAHFA families, including 262 regioisomers, in rice and Arabidopsis thaliana, but did not quantify them. Besides saturated FAHFAs, Pham et al. [41] detected many unsaturated and polyunsaturated fatty acids in caribou meat and moose at very higher doses. Polyunsaturated FAHFAs were also identified in oat oil [33], with LAHLAs being the most pronounced. FAHFAs are also present in breast milk [5], although at very low concentrations. Indeed, the mammary gland synthesizes FAHFAs during breastfeeding. Interestingly, it has been shown that 5-PAHSA as well as total PAHSA levels were significantly lower in the milk of obese compared to lean mothers [5].

\subsection{Endogenous distribution of FAHFAs and metabolic factors modulating their levels}

Saturated and unsaturated long chain fatty acid-derived FAHFAs are present in blood and in many tissues in rodent and humans, mainly in white and brown adipose tissue. Yore et al. [2] showed that FAHSA levels vary from tissue to tissue depending on the FAHSA considered, with the highest levels of PAHSAs found in white and brown adipose tissue (100 and $150 \mathrm{ng} / \mathrm{g}$ respectively in these tissues and 10-20 ng in liver, kidney and pancreas) and 9PAHSA being the most abundant isomer in these tissues. Numerous FAHFAs (mainly 13-, 12- and 9-PAHSA, 9-OAHSA and 13-, 12- and 9-SAHSA) were also detected in rat lung, kidney, thymus, liver and heart but in lower content (pg/g) [42]. As mentioned in IV.1, polyunsaturated FAHFAs were detected in caribou and moose muscle in very high content $(\mu \mathrm{g} / \mathrm{g})$ [41] and this finding may have potential significance in functional food applications. Short chain fatty acid-derived fatty acids esters (SFAHFAs) were recently identified in intestine, liver, and plasma of rats, the large intestine having the highest abundance of SFAHFAs with a concentration range from 0.84 to $57 \mathrm{pmol} / \mathrm{mg}[11,43]$.

Several studies in both rodents and humans showed that FAHFAs, in particular PAHSA, content is decreased with adipose tissue dysfunction, obesity, insulin resistance, diabetes and with aging while fasting and physical training have a beneficial effect by stimulating the production of FAHFAs.

\subsubsection{Metabolic state associated to unfavourable FAHFAs content}

Metabolic dysfunction in adipose tissue of healthy moderately overweight humans was associated with reduced levels of PAHSAs in the same tissue $[44,45]$. With obesity, insulin-resistance and diabetes, PAHSAs content were differently modulated in tissues in mice and in human: decreased PAHSA levels were observed in white fat and liver of obese and insulin-resistant mice; in serum of obese patients and in diabetics, the levels of all PAHSAs except 9PAHSA were reduced [2,46]; different alterations of FAHFAs were observed in human plasma from overweight/obese individuals of normoglycemia, as well as $\mathrm{db} / \mathrm{db}$ mouse liver and white adipose tissues compared to that in the controls [47]; SFAHFAs were decreased in tissues in high-fat diet fed rats compared to normal diet fed rat [11].

A large study carried out in C57B16 mice between 1 and 24 months of age showed that the number of FAHFAs (both family and regioisomer) in visceral adipose tissue increased with age, from 35 families ( $186 \pm 0$ regioisomers) at 1 -month-old mice to 46 families (278 \pm 6 regioisomers) in 18-month-old mice [48].

\subsubsection{Metabolic state associated to favourable FAHFAs content}

During fasting, increased PAHSA levels were observed in certain tissues of mice (subcutaneous fat, kidneys) and in their serum; this increase was abolished by re-feeding [2]. Exercise training stimulated production of insulin-sensitizing lipid mediators PAHSAs. 
Table 1

FAHFAs content in food (approximate level).

\begin{tabular}{|c|c|c|c|c|c|c|}
\hline \multirow[t]{2}{*}{ Food } & & \multicolumn{4}{|l|}{ Content } & \multirow[t]{2}{*}{ References } \\
\hline & & FAHFAs (ng/g fresh weight) & 9-PAHSA (ng/g) & 10-PAHSA (ng/g) & 13/12-PAHSA (ng/g) & \\
\hline \multirow[t]{11}{*}{ Fruit } & Clementine & 250 & & & & 38 \\
\hline & Pineapple & 215 & & & & 38 \\
\hline & Strawberry & 160 & & & & 38 \\
\hline & Mango & 150 & & & & 38 \\
\hline & Apple & 105 & 0.216 & 0.161 & 0.404 & 2,38 \\
\hline & Banana & 105 & & & & 38 \\
\hline & Cherry tomato & 90 & & & & 38 \\
\hline & Avocado & 85 & & & & 38 \\
\hline & Lemon & 65 & & & & 38 \\
\hline & Blueberry & 50 & & & & 38 \\
\hline & Kiwifruit & 45 & & & & 38 \\
\hline \multirow[t]{10}{*}{ Vegetable, cereals, plants } & Whole oat grain & 320 & & & & 38 \\
\hline & Garlic & 245 & & & & 38 \\
\hline & Carrot & 140 & & & & 38 \\
\hline & Parsley root & 115 & & & & 38 \\
\hline & Onion & 110 & & & & 38 \\
\hline & Pepper & 105 & & & & 38 \\
\hline & Coarse oat flakes & 80 & & & & 38 \\
\hline & Radish & 60 & & & & 38 \\
\hline & Broccoli & & 0.943 & 0.243 & 0.647 & 2 \\
\hline & Rice & NQ & & & & 39 \\
\hline \multirow[t]{6}{*}{ Food of animal origin } & Beef & & 0.215 & 3.23 & 3.23 & 2 \\
\hline & Chicken & & 0.647 & 1.08 & 1.08 & 2 \\
\hline & Egg white & & 0.135 & 0.108 & 0.96 & 2 \\
\hline & Egg yolk & & 0.216 & 24.3 & 3.77 & 2 \\
\hline & Caribou & 50. $10^{3}$ & & & & 40 \\
\hline & Moose & 40. $10^{3}$ & & & & 40 \\
\hline
\end{tabular}

NQ: not quantified.

Thus, resistive and aerobic training for 4 months of sedentary elderly women increased the content of PAHSA in serum and white adipose tissue as well as the content of PAHPA-TAG, a reserve form of PAHPA [37,49].

\section{Metabolism and storage of FAHFAs}

To date, only the PAHSA biosynthesis pathway has been elucidated. Fig. 2 is a proposal scheme for the biosynthesis, storage and catabolism of PAHSAs (partially adapted from Kuda et al. [5]). Biosynthesis of unsaturated FAHFAs is not elucidated; it could imply genes related to desaturation (Scd6) and to scavenging of oxidized lipids (Cd36) [5].

\subsection{Biosynthesis of FAHFAs}

Saturated FAHFAs biosynthesis occurs mostly in the white adipose tissue and is positively regulated by carbohydrate responsiveelement binding protein (ChREBP) [2]. Two precursors of synthesis, fatty acids (FA) and hydroxylated fatty acids (HFA), are reported to be involved in the synthesis of PAHPAs. The transcription factor nuclear factor erythroid-2-related factor 2 (Nrf2) which regulates the expression of antioxidant genes [50] and contributes to the anti-inflammatory process [51] may also be important in regulating the biosynthesis of PAHSAs [5]. Recently, it was suggested that some FAHFAs from the omega-3 fatty acid family, may be synthesized in liver [10]. As cytochrome P450-catalyzed transformation of PUFA may result in the formation of hydroxyl-PUFA [52] and as it was observed deregulation of cytochrome P450 family members in liver of patients with parental nutrition, associated with an elevated content of FAHFA, an involvement of cytochrome P450 in liver FAHFAs biosynthesis [53] cannot be excluded.

While our daily diet is a source of fatty acids, the latter are also biosynthetized from carbohydrates via de novo lipogenesis. De novo lipogenesis is a complex metabolic pathway mainly regulated by transcription factors such as sterol regulatory element binding protein-1c (SREBP-1c), ChREBP, liver X receptor $\alpha(\mathrm{LXR} \alpha)$, and peroxisome proliferator-activated receptor $\gamma$ (PPAR $\gamma$ ) [54-56].

The hydroxy saturated fatty acids are formed by oxidation of fatty acids from phospholipids [57]. Membrane phospholipids can be oxidized by radical oxygen species to phospholipid hydroperoxides, or phospholipid precursors can be synthesized via de novo lipogenesis and enzymatically oxidized. Phospholipid hydroperoxides can be reduced by enzymes with GSH peroxidase or transferase activity (Peroxiredoxin-6 (Prdx6), Microsomal Glutathione STransferase 1 and 3 (Mgst1 andMgst3) to a phospholipid with a hydroxylated acyl chain. This reaction is dependent on cellular redox state and probably controlled via an Nrf2 master regulator. The hydroxy fatty acid is usually cleaved through an oxidized phospholipid remodeling process (probably via Prdx6 that possess a phospholipase A2 acyltransferase activity) [5].

It has been reported [5] that hydroxy fatty acids may be esterified with acyl-CoA fatty acids by acyltransferase (probably via Bile Acid-CoA:Amino Acid N-Acyltransferase (Baat) and Acyl-coenzyme A amino acid N-acyltransferase 1 and 2 (Acnat1 and Acnat2)). Moreover, detection of a higher quantity of the (R)-isomer than the (S)-isomer [18] suggests an enzyme-catalyzed stereospecific biosynthesis of 9-PAHSA.

\subsection{Storage of FAHFAs}

The discovery of a structurally novel class of triacylglycerols which contain a FAHFA in place of one of the three fatty acids was recently reported [37]. The concentration of FAHFA-TAGs in white adipose tissue is 100 times higher than that of free FAHFAs. Triacylglycerols allow the storage of fatty acids within tissues, particularly in adipose tissues, in postprandial periods, and the FAHFA of triacylglycerols (FAHFA-TAGs) are believed to be an 


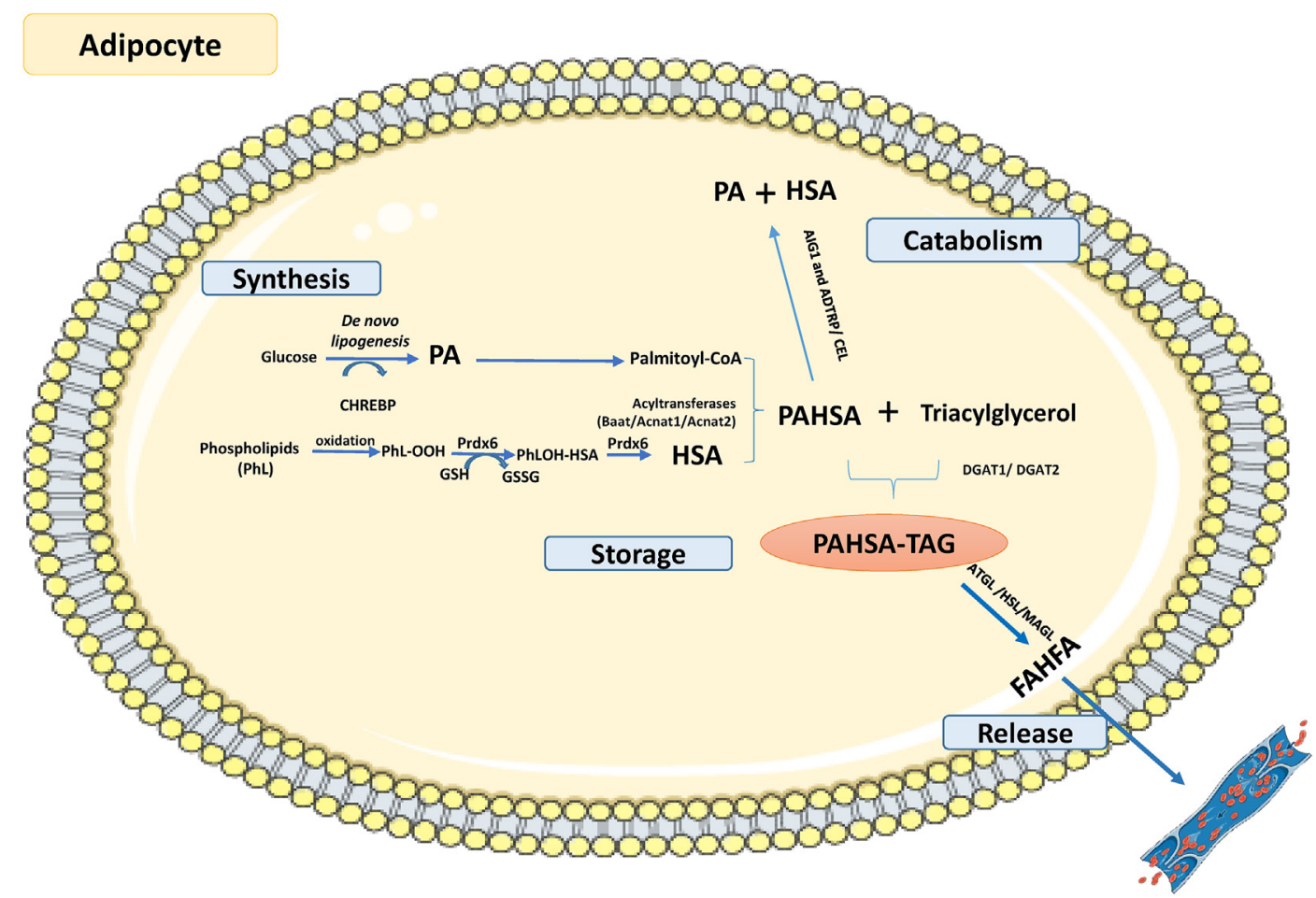

Fig. 2. Metabolism of PAHSA in adipocytes.

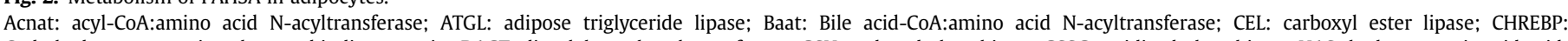

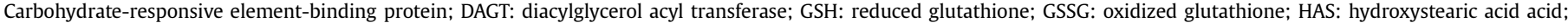

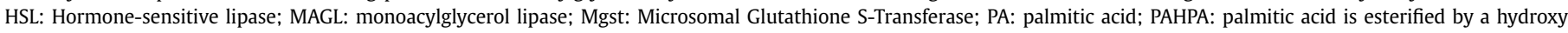
stearic acid; PhL: phospholipid; PhL-OOH: phospholipid hydroperoxides; PhLOH: phospholipid with a hydroxylated acyl chain; Prdx6: Peroxiredoxin-6.

Fig. 2 is a proposal scheme for the biosynthesis and metabolism of PAHSAs (partially adapted from Kuda et al. [5]).

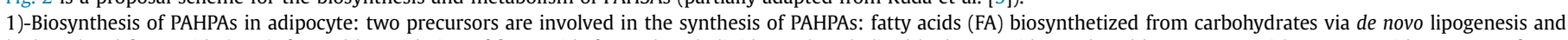

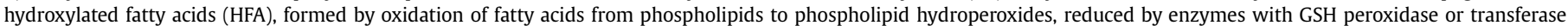

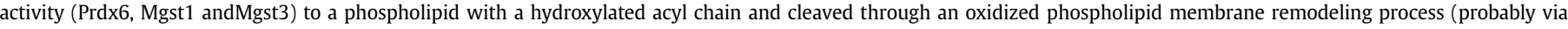
Prdx6 that possesses a phospholipase A2 acyltransferase activity) [5]. Hydroxy fatty acids may then be esterified with acyl-CoA fatty acids by an acyltransferase.

2)-Storage of FAHFAs in adipocyte: it occurs mainly as FAHFA-TAGs, with the involvement of diglyceride acyltransferases DGAT1 and DGAT2 enzymes.

3)-Catabolism of FAHFAs: Two categories of enzymes degrade FAHFAs, namely Ser/Thr hydrolases (AIG1 and ADTRP) [88] and CEL [61].

important reservoir of FAHFAs in cells and tissues.

The FAHFA moiety is hydrolyzed under fasting conditions to release a non-esterified fatty acid, which can be used as a fuel to fulfill the energy requirements or serve as signalling molecules. The regulated balance of fatty acid esterification and triacylglycerols hydrolysis provides a buffer system to maintain physiological levels of fatty acids. Data from Tan et al. [37] suggest that FAHFA-TAGs may play a similar role in regulating the body's FAHFA levels.

The enzymes involved in the synthesis of FAHFA-TAGs are the two key enzymes involved in the synthesis of triacylglycerols, namely the diacylglycerol acyltransferases DGAT1 and DGAT2 [58]. Indeed Tan et al. [37] have shown that FAHFA-TAGs levels are reduced when DGAT1 and DGAT2 are inhibited in adipocytes in vitro. Interestingly, a high-fat diet increased the FAHFA-TAGs content of adipose tissue in mice.

The enzymes responsible for the release of FAHFAs may be the same enzymes as for the hydrolysis of triacylglycerols, i.e. adipose triacylglycerol lipase (ATGL), hormone-sensitive lipase (HSL) and monoacylglycerol lipase (MAGL) [17,37,59].

To date, it is not established whether FAHFAs and FAHFA-TAGs are co-regulated or differentially regulated in different metabolic states. It also remains to be determined whether FAHFA-TAGs are only a reservoir of FAHFAs in cells and tissues or whether they have other cellular functions.

\subsection{Catabolism of FAHFAs}

Two categories of enzymes, namely Ser/Thr hydrolases (AIG1 and ADTRP) [60] and carboxyl ester lipase (CEL) [61] degrade FAHFAs. The enzymes AIG1 (androgen-induced gene 1 protein) and ADTRP (Androgen Dependent TFPI Regulating Protein) are a family of atypical integral membrane hydrolases that catabolize FAHFAs [60]. The cell/tissue distributions of these enzymes are quite distinct, with AIG1 being predominantly ubiquitously expressed, and ADTRP having a more restricted expression profile, mainly in metabolic organs such as liver, kidney, gut and adipose tissue (white and brown) [62]. ADTRP is the major hydrolase in white and brown adipose tissue. However, functional differences between AIG1 and ADTRP have not been demonstrated.

The lipolytic enzyme CEL, previously called cholesterol esterase, is synthesized primarily in the acinar cells of the exocrine pancreas [63]. Recently, it was reported that CEL is capable of hydrolysing FAHFAs [61]. It was also demonstrated that CEL has preferences among different FAHFAs family, FAHFAs regioisomers and among enantiomers, with a preference for 9(S)-PAHSA [18], indicating the regio- and stereoselectivity of the enzyme. The complementary tissue distribution of CEL compared to AIG1 and ADTRP hydrolases indicates that the metabolism of FAHFAs could be differentially regulated across the body [61]. 


\section{Beneficial effects of FAHFAS in the obesity-related metabolic disorders}

Obesity increases risks for many chronic degenerative diseases, particularly insulin resistance and type 2 diabetes mellitus and is generally associated with chronic low grade inflammation in a variety of tissues [64]. Oxidative stress can aggravate metabolic complications in an obese state [65]. Anti-diabetic and antiinflammatory activity of FAHFAs therefore may have beneficial effects in obesity. To date, as discussed below, the PAHSA family is the most studied. However, the effects of omega-3 fatty acid FAHFAs (DHAHLA and EPAHSA families) [4,32], omega-6 fatty acid derived FAHFAs (several LAHLAs) [66], and two FAHFAs differing only in the length and saturation of the fatty acid chain (9-PAHPA and 9OAHPA) $[12,13,67]$ were also investigated.

\subsection{FAHFAs have beneficial effects in insulin resistance and diabetes}

As discussed below, FAHFAs may have direct and indirect effects on improving glucose tolerance and insulin sensitivity. Several studies have been conducted in cellular and animal models of obesity and/or diabetes.

\subsubsection{FAHFAs improve glucose metabolism and insulin signalling}

Several studies in adult C57BL6/J mice consuming a control diet or a high-fat obesogenic diet with insulin resistance have shown that acute oral administration or chronic subcutaneous administration of 5- or 9-PAHSA improved insulin sensitivity and glucose tolerance $[2,15,68]$. This improvement was associated with an increase in serum PAHSA levels in these animals. Interestingly, levels of these FAHFAs were reported to be elevated in adipose tissue and serum in transgenic AG4OX mice over-expressing glucose transporter 4 (GLUT4) associated with an increased glucose tolerance compared to control C57B6/J mice [2,46,68].

In adipose tissues, 9-PAHSA has been shown to improve insulin sensitivity by an increase in the glucose transport (GLUT4) and its translocation from intracellular vesicles to the plasma membrane $[2,46]$.

Interestingly, it has been recently reported [69] that chronic administration of 5- or 9-PAHSA in C57BL6/j mice fed a high-fat diet increased glucose absorption in EDL muscle (long extensor muscle) but not in other glycolytic muscles or in the heart, by promoting AKT signalling in this tissue (cell signalling kinase). 9-PAHSA administration also improved insulin sensitivity in the liver by activating the p-AKT pathway, which increased liver glycogen levels in these mice and reduced glucose production by the liver [69]. In addition, acute intravenous administration of 9-PAHSA decreased endogenous glucose production by the liver by inhibiting the cAMP/PKA signalling pathway, resulting in decreased activity of glucose-6-phosphatase (G6pase) and phosphoe nolpyruvate carboxykinase 1 (Pck1). Furthermore, 5- or 9-PAHSAs indirectly increased hepatic insulin sensitivity by inhibiting lipolysis and lowering serum free fatty acid levels [69].

In a recent paper, Syed et al. [70] have tested the influence of 5and 9-PAHSA in an NOD (non-obese diabetic) mouse model of autoimmune diabetes (type 1). Chronic treatment with 5- and 9PAHSA protected against the development of type 1 diabetes in these NOD mice, increased serum insulin levels in these mice and delayed the onset of diabetes. In addition, 5- and 9-PAHSA attenuated the immune response that causes type 1 diabetes in this model and promoted $\beta$-cell survival [70].

In mice fed with a control diet or supplemented with 9-PAHPA or 9-OAHPA for 12 weeks, both these FAHFAs increased basal metabolism and insulin sensitivity, but without modifying glucose tolerance [13]. Our data also showed that this insulin-sensitive effect was probably exerted at the skeletal muscle level [67]. However, 9-PAHPA intake and to a lesser extent 9-OAHPA intake induced liver steatosis and fibrosis in some healthy mice. It is likely that both FAHFAs have insulin-sensitized the healthy liver so much that de novo lipogenesis promoted steatosis/fibrosis. 9-PAHPA and 9-OAHPA also modulated basal metabolism and improved insulin sensitivity in obesogenic diet-fed mice [12], without induced hepatic alteration in contrast to that observed in the healthy mice. In vitro, a therapeutic effect of 9-PAHSA on lipotoxicity in steatotic primary hepatocytes and HepG 2 cells was observed, but additional investigation is required to elucidate the 9-PAHSA impact on hepatosteatosis and NASH [71].

In vitro, 5-PAHSA has been shown to directly enhance glucosestimulated insulin secretion in islets of human pancreatic $\beta$-cells [2]. In addition, chronic incubation (48h) with 5-PAHSA of pancreatic islets of Langerhans isolated from male $C D-1$ type mice (Swiss mouse strain) and pancreatic islets from healthy or diabetic patients significantly increased glucose-stimulated insulin secretion [3].

\subsubsection{FAHFAs activate GPR120 and GPR40 and increase GLP-1 secretion}

The G protein-coupled receptor 120 (GPR120) regulates glycemic homeostasis via its ability to stimulate the secretion of Glucagon-Like Peptide 1 (GLP-1, an incretin with insulinotropic activity) and Cholecystokinin (CCK, an intestinal enzyme that stimulates insulin secretion and modulates the feeling of satiety) following its activation by unsaturated fatty acids [72]. Regioisomers 5- and 9-PAHSA increased the expression of GPR120 and GLUT4 and the translocation of GLUT4 in adipocyte lines 3T3L1 [2,44]. Recently, it was shown that 5- and 9-PAHSA also activates $G$ protein-coupled receptor 40 (GPR40), a fatty acid receptor of the same family as GPR120 [73].

5- or 9-PAHSA also stimulates insulin secretion in response to glucose via increased GLP-1 secretion. GLP-1 is an incretin that modulates carbohydrate homeostasis by stimulating insulin secretion and inhibiting the secretion of glucagon $[2,15,68,74]$.

\subsubsection{FAHFAs attenuate diabetic-induced cognitive dysfunction}

Finally, administration of 9-PAHSA in diabetic mice decreased repressor element-1 silencing transcription factor/neuronrestrictive silence factor and upregulated brain-derived neurotrophic factor expression in frontal cortex; thus 9-PAHSA attenuated cognitive impairment [75].

\subsection{FAHFAs have beneficial effects in inflammation}

As discussed below, several in vitro and in vivo studies have shown that some saturated and polyunsaturated FAHFAs have antiinflammatory effects, notably DHAHLA omega-3 FAHFAs. Due to the role of inflammation in obesity, targeting inflammation represents an important issue for the prevention of obesity-linked diseases, including insulin resistance and type 2 diabetes [64].

\subsubsection{FAHFAs reduce chronic inflammation}

In vivo, Yore et al. [2] showed that administration of 9-PAHSA to mice consuming a high-fat diet reduced inflammation in adipose tissue by reducing macrophage production of two proinflammatory interleukins, IL- 1 and Tumor Necrosis Factor $\alpha$ (TNF $\alpha$ ), but this effect was not observed with 5-PAHSA suggesting that individual family members in a FAHFA family can have distinct activities or different potencies for the same activity. In addition, three days of gavage with 5- and 9-PAHSA in mice consuming a high-fat diet reduced the percentage of GAB [2], a protein that controls the function of several receptors and expresses pro- 
inflammatory cytokines, growth factors and antigen receptors on $\mathrm{T}$ and B cells [76,77]. In vitro, on a cell line from the vascular stromal fraction of adipose tissue, Yore et al. [2] showed that chronic treatment with 5- and 9- PAHSA reduced the total number of AT CD11c macrophages (macrophages secreting the CD11c monocyte differentiation marker).

\subsubsection{FAHFAs reduce acute inflammation}

In vivo, Lee et al. [78] documented in a mouse model of ulcerative colitis that treatment with 9-PAHSA reduces disease severity through direct inhibition of dendritic cell activation and subsequent polarization of $\mathrm{T}$ helper type 1 cells. In a rat model of knee osteoarthritis (KOA) [79], the serum concentration of FAHFAs decreased and application of a "Sanse powder" (patch with Chinese medicinal herbs) not only had a significant protective effect against KOA but also restored serum levels of FAHFAs.

In vitro, Yore et al. [2] showed that 9-PAHSA blocked the effect of lipopolysaccharide (LPS) on the activation of bone marrow-derived dendritic cells and the resulting production of pro-inflammatory cytokines such as interleukin IL-12, IL-1 $\beta$ and TNF $\alpha$. Reduction of the carbon chain lengths of the 9-hydroxystearic acid portion or palmitic acid hydrocarbon chain resulted in lower molecular weight analogues that maintained anti-inflammatory activity (attenuation of IL-6 production) in the murine macrophage cell line RAW 264.7 treated with LPS [9]. Moreover, Kuda et al. [4] showed in primary mouse bone marrow-derived macrophage cells and/or in macrophage cell lines (RAW 264.7) that 9-PAHSA and 13-DHAHLA inhibited LPS-induced secretion of pro-inflammatory cytokines such as IL-6, IL- $1 \beta$ and TNF- $\alpha$. In addition, 13-DHAHLA decreased the activation of indoleamine 2,3-dioxygenase in leukocytes, an enzyme that exerts an immunosuppressive effect. Kolar et al. showed that 13-LAHLA suppressed LPS-stimulated secretion of cytokines and expression of pro-inflammatory genes in macrophage cell lines (RAW 264.7) [33]. These authors also reported [33] that polyunsaturated FAHFAs are more potent anti-inflammatory agent than saturated FAHFAs.

\subsection{FAHFAs have others beneficial health effects}

\subsubsection{FAHFAs may promote browning of white adipose tissue}

Recently, it was shown [80] that 9-PAHSA not only inhibited LPS-induced inflammation in 3T3-L1 cells but also promoted 3T3L1 cells browning through GPR120; also, 9-PAHSA induced browning of white adipose tissue in wild-type and ob/ob mice. Wang et al. [80] demonstrated that the anti-inflammatory role of 9PAHSA may be beneficial in treating obesity not only directly by lowering inflammation but also indirectly, as browning of white adipose tissue favour its thermogenic activity and prevent excess lipid accumulation.

\subsubsection{FAHFAs may have antioxidant effects}

A recent study suggested that EPA-derived FAHFAs, a family with omega-3 fatty acid derived-FAHFAs, were strong Nrf2 activators with plausible antioxidant effects. It was shown that they suppressed the oxidation of small lipids droplets, oxidative stress induced by $\mathrm{H}_{2} \mathrm{O}_{2}$ and enhanced the gene expression of cytoprotective antioxidant enzymes $(\mathrm{NAD}(\mathrm{P}) \mathrm{H}$ dehydrogenase Quinone 1 (NQO1), Glutamate-Cysteine Ligase Modifier Subunit (GCLM), Superoxide Dismutase (SOD-1), Heme Oxygenase (HO-1)) [10]. These observations are of great significance for the treatment of oxidative stress-related disorders.

\subsubsection{FAHFAs may have beneficial effects against cancer} development

Lee et al. [78] observed that 9-PAHSA reduced the disease severity in a mouse model of ulcerative colitis. As patients with ulcerative colitis are at higher risk for developing colorectal cancer than the general population [81], this suggests a favourable effect of FAHFAs against cancer development. Lower plasma levels of FAHFAs (13-, 9-PAHSA, 13- and 12-SAHSA) were also observed in patients with breast cancer [42]. Recently, Rodriguez et al. [82] showed that 9-HSA-containing FAHFA levels (9-SAHSA, 9-PAHSA and 9-OAHSA) in colorectal tumors were elevated compared with normal tissue. Importantly, in vitro studies, these FAHFAs did not induce apoptosis of colorectal cell lines (HT-29 and HCT-116) unlike what was observed with the hydroxy fatty acid 9-HAS alone. Furthermore, the addition of 9-HSA to colon cancer cell lines augmented the synthesis of different FAHFAs before the cells commit to apoptosis. These observations suggested that FAHFA formation may function as a buffer system that sequesters the hydroxyl-acid into an inactive form, thereby restricting apoptosis. Rodriguez et al. [82] proposed that FAHFA synthesis may occur at proliferative tumor stages where there is a predominance of mitosis over apoptosis and, in opposition, cells may activate FAHFA hydrolysis at times of declining cell death, thus regulating the cell cycle via lipid signalling. Thus, in addition to its well described roles as anti-diabetic and anti-inflammatory lipids, FAHFA may be produced as an anti-apoptotic strategy to improve the pro-apoptotic effects of hydroxy fatty acids accumulating in cells.

\subsection{Some studies do not find the described effects on glucose metabolism and inflammation}

The $\mathrm{db} / \mathrm{db}$ mice, having a defect in the $(\mathrm{db})$ gene encoding the leptin receptor and characterized by obesity and glucose intolerance, constitute a well-known model of type 2 diabetes. In $\mathrm{db} / \mathrm{db}$ mice, Wang et al. [83] found that oral administration of 5-PAHSA for 1 month had no beneficial effect on glucose metabolism. Administration of 5-PAHSA did not reduce the observed hyperglycemia and did not improve insulin sensitivity and glucose tolerance. On the contrary, increased inflammation and aggravation of hepatic steatosis were observed in these mice.

Another team also found no effect of acute or chronic treatment with 5- and 9-PAHSA on glucose metabolism [27]. In vitro studies using human adipocytes, rat muscle cells (L6) and mouse enteroendocrine cell lines or rat and human pancreatic islets, treatment with 5- and 9-PAHSA did not affect glucose uptake, insulin secretion or GLP-1 release at any concentration of glucose from the medium. In vivo studies using different diets rich in obesityinducing fats were explored in mice (beef tallow, lard, vegetable oil and coconut oil) and treatment with 5- and 9-PAHSA in these mice did not improve the general metabolic status nor did it improve glucose absorption, insulin secretion or GLP-1 secretion. However, limitations were highlighted $[84,85]$ in the work of Pflimlin et al. [27], in particular concerning the use of olive oil as a vehicle for PAHSA. Olive oil, which contains many bioactive and metabolically beneficial molecules, including omega- 3 and -6 fatty acids, tocopherols, polyphenols, flavones and even FAHFAs, could have masked the proper effect of PAHSA on glucose homeostasis and GLP-1 secretion. Furthermore, Pflimlin et al. [27] studied the in vitro effects of PAHSA on glucose transport in human cells using maximal insulin concentrations, conditions under which it would be impossible to detect the insulin-sensitizing effects of PAHSA according to Syed et al. [84].

In addition, a recent work raised the possibility that FAHFAs are markers rather than mediators of insulin sensitivity, at least in Bcl6 AKO mice [86]. In fact in Bcl6 AKO mice (KO model for adipocyte $\mathrm{Bcl6}$, a transcriptional repressor) [87], Senagolage et al. [86] observed an increase in de novo lipogenesis and an expansion of subcutaneous white adipose tissue, in association with protection 
against obesity-induced insulin resistance and with high levels of FAHFAs. However, it is difficult to conclude in this sense on the basis of this study alone.

\section{Conclusion}

FAHFAs are a new class of recently discovered endogenous lipids whose the tissue levels are reduced in obesity and type 2 diabetes. Moreover, some of these molecules, produced de novo in some tissues, improve glucose tolerance and insulin sensitivity and stimulate insulin secretion, and thus may have beneficial effects against insulin resistance and type 2 diabetes. Anti-inflammatory effects of some FAHFAs have also been reported, both with acute and chronic inflammation states. Other beneficial health effects have been also suggested, in particular against some cancers So, FAHFAs could therefore be a potential therapeutic target for the treatment of numerous metabolic disorders, however a lot of research in humans is still necessary to validate this possibility. Beforehand it will also be important to determine the synthesis and degradation pathways of these molecules in the body. In addition, the studies aiming at investigating the health/metabolic effects of FAHFAs on liver and skeletal muscle should be continued and expanded. Indeed, these two organs are crucial in the prevention or development of metabolic diseases.

\section{Funding}

Melha Benlebna thanks the Algerian Ministry of Higher Education and Scientific Research for the financial support of her PhD program.

\section{Declaration of competing interest}

The authors declare that they have no known competing financial interests or personal relationships that could have appeared to influence the work reported in this paper.

\section{Acknowledgement}

The authors would like to thank Jonathan Levin for the careful reading of the manuscript and for improving the use of English.

\section{References}

[1] K.G. Alberti, P. Zimmet, J. Shaw, I. D. F. E. T. F. C. Group, The metabolic syndrome-a new worldwide definition, Lancet 366 (2005) 1059-1062.

[2] M.M. Yore, I. Syed, P.M. Moraes-Vieira, T. Zhang, M.A. Herman, E.A. Homan, R.T. Patel, J. Lee, S. Chen, O.D. Peroni, A.S. Dhaneshwar, A. Hammarstedt, U. Smith, T.E. McGraw, A. Saghatelian, B.B. Kahn, Discovery of a class of endogenous mammalian lipids with anti-diabetic and anti-inflammatory effects, Cell 159 (2014) 318-332.

[3] B. Bandak, L. Yi, M.G. Roper, Microfluidic-enabled quantitative measurements of insulin release dynamics from single islets of Langerhans in response to 5palmitic acid hydroxy stearic acid, Lab Chip 18 (2018) 2873-2882.

[4] O. Kuda, M. Brezinova, M. Rombaldova, B. Slavikova, M. Posta, P. Beier, P. Janovska, J. Veleba, J. Kopecky Jr., E. Kudova, T. Pelikanova, J. Kopecky, Docosahexaenoic acid-derived fatty acid esters of hydroxy fatty acids (FAHFAs) with anti-inflammatory properties, Diabetes 65 (2016) 2580-2590.

[5] O. Kuda, M. Brezinova, J. Silhavy, V. Landa, V. Zidek, C. Dodia, F. Kreuchwig, M. Vrbacky, L. Balas, T. Durand, N. Hubner, A.B. Fisher, J. Kopecky, M. Pravenec, Nrf2-Mediated antioxidant defense and Peroxiredoxin 6 are linked to biosynthesis of palmitic acid ester of 9-hydroxystearic acid, Diabetes 67 (2018) 1190-1199.

[6] L. Balas, C. Feillet-Coudray, T. Durand, Branched fatty acyl esters of hydroxyl fatty acids (FAHFAs), appealing beneficial endogenous fat against obesity and type-2 diabetes, Chemistry 24 (2018) 9463-9476.

[7] M.H. Kaspersen, L. Jenkins, J. Dunlop, G. Milligan, T. Ulven, Succinct synthesis of saturated hydroxy fatty acids and in vitro evaluation of all hydroxylauric acids on FFA1, FFA4 and GPR84, Medchemcomm 8 (2017) 1360-1365.

[8] A.T. Nelson, Synthesis, SAR Library, and Chemical Biology Probes of PAHSAs, a Family of Natural Product Lipids with Anti-diabetic Activity, University of
Texas at Austin, 2018. Dissertation: Doctor of Phylosophy, University of Texas at Austin - Dissertation: Doctor of Phylosophy.

[9] H. Wang, T. Chang, S. Konduri, J. Huang, A. Saghatelian, D. Siegel, Synthesis of chemically edited derivatives of the endogenous regulator of inflammation 9PAHSA, J. Antibiot. (Tokyo) 72 (2019) 498-506.

[10] S.G.B. Gowda, H. Fuda, T. Tsukui, H. Chiba, S.P. Hui, Discovery of eicosapentaenoic acid esters of hydroxy fatty acids as potent Nrf2 activators, Antioxidants 9 (5) (2020) 397.

[11] S.G.B. Gowda, C. Liang, D. Gowda, F. Hou, K. Kawakami, S. Fukiya, A. Yokota, H. Chiba, S.P. Hui, Identification of short chain fatty acid esters of hydroxy fatty acids (SFAHFAs) in murine model by nontargeted analysis using ultrahigh-performance liquid chromatography/linear trap quadrupole-Orbitrap mass spectrometry, Rapid Commun. Mass Spectrom. 34 (17) (2020), e8831.

[12] M. Benlebna, L. Balas, B. Bonafos, L. Pessemesse, G. Fouret, C. Vigor, S. Gaillet, J. Grober, F. Bernex, J.F. Landrier, O. Kuda, T. Durand, C. Coudray, F. Casas, C. Feillet-Coudray, Long-term intake of 9-PAHPA or 9-OAHPA modulates favorably the basal metabolism and exerts an insulin sensitizing effect in obesogenic diet-fed mice, Eur. J. Nutr. (2020 Sep 28).

[13] M. Benlebna, L. Balas, B. Bonafos, L. Pessemesse, C. Vigor, J. Grober, F. Bernex, G. Fouret, V. Paluchova, S. Gaillet, J.F. Landrier, O. Kuda, T. Durand, C. Coudray, F. Casas, C. Feillet-Coudray, Long-term high intake of 9-PAHPA or 9-OAHPA increases basal metabolism and insulin sensitivity but disrupts liver homeostasis in healthy mice, J. Nutr. Biochem. 79 (2020) 108361.

[14] D. Rychtrmoc, P. Stankova, O. Kucera, Z. Cervinkova, Comparison of two antidiabetic monoestolides regarding effects on intact murine liver tissue, Arch. Physiol. Biochem. 25 (2020) 1-8.

[15] I. Syed, J. Lee, P.M. Moraes-Vieira, C.J. Donaldson, A. Sontheimer, P. Aryal, K. Wellenstein, M.J. Kolar, A.T. Nelson, D. Siegel, J. Mokrosinski, I.S. Farooqi, J.J. Zhao, M.M. Yore, O.D. Peroni, A. Saghatelian, B.B. Kahn, Palmitic acid hydroxystearic acids activate GPR40, which is involved in their beneficial effects on glucose homeostasis, Cell Metabol. 27 (2018), 419-427 e414.

[16] A. Vijayakumar, P. Aryal, J. Wen, I. Syed, R.P. Vazirani, P.M. Moraes-Vieira, J.P. Camporez, M.R. Gallop, R.J. Perry, O.D. Peroni, G.I. Shulman, A. Saghatelian, T.E. McGraw, B.B. Kahn, Absence of carbohydrate response element binding protein in adipocytes causes systemic insulin resistance and impairs glucose transport, Cell Rep. 21 (2017) 1021-1035.

[17] V. Paluchova, M. Oseeva, M. Brezinova, T. Cajka, K. Bardova, K. Adamcova, P. Zacek, K. Brejchova, L. Balas, H. Chodounska, E. Kudova, R. Schreiber, R. Zechner, T. Durand, M. Rossmeisl, N.A. Abumrad, J. Kopecky, O. Kuda, Lipokine 5-PAHSA is regulated by adipose triglyceride lipase and primes adipocytes for de novo lipogenesis in mice, Diabetes 69 (2020) 300-312.

[18] A.T. Nelson, M.J. Kolar, Q. Chu, I. Syed, B.B. Kahn, A. Saghatelian, D. Siegel, Stereochemistry of endogenous palmitic acid ester of 9-hydroxystearic acid and relevance of absolute configuration to regulation, J. Am. Chem. Soc. 139 (2017) 4943-4947.

[19] H. Wang, M.J. Kolar, T. Chang, J. Rizo, S. Konduri, C. McNerlin, A. Saghatelian, D. Siegel, Stereochemistry of linoleic acid esters of hydroxy linoleic acids, Org. Lett. 21 (2019) 8080-8084.

[20] A. Vik, T.V. Hansen, O. Kuda, Synthesis of both enantiomers of the docosahexaenoic acid ester of 13-hydroxyoctadecadienoic acid (13-DHAHLA), Tetrahedron Lett. 60 (2019) 151331.

[21] D.B. Weibel, L.E. Shevy, F.C. Schroeder, J. Meinwald, Synthesis of mayolene-16 and mayolene-18: larval defensive lipids from the European cabbage butterfly, J. Org. Chem. 67 (2002) 5896-5900.

[22] I. Uchida, K. Yoshida, Y. Kawai, S. Takase, Y. Itoh, H. Tanaka, M. Kohsaka, H. Imanaka, Studies on WB-3559 A, B, C and D, new potent fibrinolytic agents. II. Structure elucidation and synthesis, J. Antibiot. (Tokyo) 38 (1985) $1476-1486$.

[23] I. Uchida, K. Yoshida, Y. Kawai, S. Takase, Y. Itoh, H. Tanaka, M. Kohsaka, H. Imanaka, Structure and synthesis of WB-3559 A, B, C and D, new fibrinolytic agents isolated from Flavobacterium sp, Chem. Pharm. Bull. (Tokyo) 33 (1985) 424-427.

[24] M. Shiozaki, N. Deguchi, T. Mochizuki, T. Wakabayashi, T. Ishikawa, H. Haruyama, Y. Kawai, M. Nishijima, Revised structure and synthesis of flavolipin, Tetrahedron 54 (1998) 11861-11876.

[25] O.G. Mountanea, D. Limnios, M.G. Kokotou, A. Bourboula, G. Kokotos, Asymmetric synthesis of saturated hydroxy fatty acids and fatty acid esters of hydroxy fatty acids, Eur. J. Org Chem. 2019 (2019) 2010-2019.

[26] A. Bourboula, D. Limnios, M.G. Kokotou, O.G. Mountanea, G. Kokotos, Enantioselective organocatalysis-based synthesis of 3-hydroxy fatty acids and fatty gamma-lactones, Molecules 24 (11) (2019) 2081.

[27] E. Pflimlin, M. Bielohuby, M. Korn, K. Breitschopf, M. Lohn, P. Wohlfart, A. Konkar, M. Podeschwa, F. Barenz, A. Pfenninger, U. Schwahn, T. Opatz, M. Reimann, S. Petry, N. Tennagels, Acute and repeated treatment with 5PAHSA or 9-PAHSA isomers does not improve glucose control in mice, Cell Metabol. 28 (2018) 217-227.

[28] P.K. Jadhav, Asymmetric-synthesis of (3r)-alkanoyloxytetradecanoic acidscomponents of bacterial lipopolysaccharides, Tetrahedron Lett. 30 (1989) 4763-4766.

[29] F. Asaro, S. Drioli, C. Forzato, P. Nitti, An efficient synthesis of chiral nonracemic hydroxyalkanoic acids by olefin cross-metathesis reactions, Chemistry 3 (2018) 13372-13376.

[30] C. Dietz, T.K. Hart, R. Nemati, X. Yao, F.C. Nichols, M.B. Smith, Structural verification via convergent total synthesis of dipeptide-lipids isolated from Porphyromonas gingivalis, Tetrahedron 72 (2016) 7557-7569. 
[31] M. Brezinova, O. Kuda, J. Hansikova, M. Rombaldova, L. Balas, K. Bardova, T. Durand, M. Rossmeisl, M. Cerna, Z. Stranak, J. Kopecky, Levels of palmitic acid ester of hydroxystearic acid (PAHSA) are reduced in the breast milk of obese mothers, Biochim. Biophys. Acta Mol. Cell Biol. Lipids 1863 (2018) $126-131$.

[32] V. Paluchova, A. Vik, T. Cajka, M. Brezinova, K. Brejchova, V. Bugajev, L. Draberova, P. Draber, J. Buresova, P. Kroupova, K. Bardova, M. Rossmeisl, J. Kopecky, T.V. Hansen, O. Kuda, Triacylglycerol-rich oils of marine origin are optimal nutrients for induction of polyunsaturated docosahexaenoic acid ester of hydroxy linoleic acid (13-DHAHLA) with anti-inflammatory properties in mice, Mol. Nutr. Food Res. 64 (2020), e1901238.

[33] M.J. Kolar, S. Konduri, T. Chang, H. Wang, C. McNerlin, L. Ohlsson, M. Harrod, D. Siegel, A. Saghatelian, Linoleic acid esters of hydroxy linoleic acids are antiinflammatory lipids found in plants and mammals, J. Biol. Chem. 294 (2019) 10698-10707.

[34] R.A. Moreau, D.C. Doehlert, R. Welti, G. Isaac, M. Roth, P. Tamura, A. Nunez, The identification of mono-, di-, tri-, and tetragalactosyl-diacylglycerols and their natural estolides in oat kernels, Lipids 43 (2008) 533-548.

[35] M. Hamberg, E. Liepinsh, G. Otting, W. Griffiths, Isolation and structure of a new galactolipid from oat seeds, Lipids 33 (1998) 355-363.

[36] S. McLean, N.W. Davies, D.S. Nichols, B.J. McLeod, Triacylglycerol estolides, a new class of mammalian lipids, in the paracloacal gland of the brushtail possum (Trichosurus vulpecula), Lipids 50 (2015) 591-604.

[37] D. Tan, M.E. Ertunc, S. Konduri, J. Zhang, A.M. Pinto, Q. Chu, B.B. Kahn, D. Siegel, A. Saghatelian, Discovery of FAHFA-containing triacylglycerols and their metabolic regulation, J. Am. Chem. Soc. 141 (2019) 8798-8806.

[38] M.G. Kokotou, Analytical methods for the determination of fatty acid esters of hydroxy fatty acids (FAHFAs) in biological samples, plants and foods, Biomolecules 10 (8) (2020) 1092.

[39] A.M. Liberati-Cizmek, M. Bilus, A.L. Brkic, I.C. Baric, M. Bakula, A. Hozic, M. Cindric, Analysis of fatty acid esters of hydroxyl fatty acid in selected plant food, Plant Foods Hum. Nutr. 74 (2019) 235-240.

[40] Q.F. Zhu, J.W. Yan, T.Y. Zhang, H.M. Xiao, Y.Q. Feng, Comprehensive screening and identification of fatty acid esters of hydroxy fatty acids in plant tissues by chemical isotope labeling-assisted liquid chromatography-mass spectrometry, Anal. Chem. 90 (2018) 10056-10063.

[41] T.H. Pham, N.P. Vidal, C.F. Manful, T.A. Fillier, R.P. Pumphrey, K.M. Doody, R.H. Thomas, Moose and caribou as novel sources of functional lipids: fatty acid esters of hydroxy fatty acids, Diglycerides and Monoacetyldiglycerides, Molecules 24 (2019) 232-247.

[42] Q.F. Zhu, J.W. Yan, Y. Gao, J.W. Zhang, B.F. Yuan, Y.Q. Feng, Highly sensitive determination of fatty acid esters of hydroxyl fatty acids by liquid chromatography-mass spectrometry, J. Chromatogr. B Analyt. Technol. Biomed. Life Sci. (2017) 34-40, 1061-1062.

[43] B.G. SG, D. Gowda, C. Liang, Y. Li, K. Kawakami, S. Fukiya, A. Yokota, H. Chiba, S.P. Hui, Chemical labeling assisted detection and identification of short chain fatty acid esters of hydroxy fatty acid in rat colon and cecum contents, Metabolites 10 (10) (2020) 398.

[44] A. Hammarstedt, I. Syed, A. Vijayakumar, B. Eliasson, S. Gogg, B.B. Kahn, U. Smith, Adipose tissue dysfunction is associated with low levels of the novel Palmitic Acid Hydroxystearic Acids, Sci. Rep. 8 (2018) 15757.

[45] A. Hammarstedt, T.E. Graham, B.B. Kahn, Adipose tissue dysregulation and reduced insulin sensitivity in non-obese individuals with enlarged abdominal adipose cells, Diabetol. Metab. Syndrome 4 (2012) 42.

[46] P.M. Moraes-Vieira, A. Saghatelian, B.B. Kahn, GLUT4 expression in adipocytes regulates de novo lipogenesis and levels of a novel class of lipids with antidiabetic and anti-inflammatory effects, Diabetes 65 (2016) 1808-1815.

[47] C. Hu, M. Wang, Q. Duan, X. Han, Sensitive analysis of fatty acid esters of hydroxy fatty acids in biological lipid extracts by shotgun lipidomics after one-step derivatization, Anal. Chim. Acta 1105 (2020) 105-111.

[48] Q.F. Zhu, J.W. Yan, J. Ni, Y.Q. Feng, FAHFA footprint in the visceral fat of mice across their lifespan, Bba-Mol. Cell Biol. Lipids 1865 (5) (2020) 158639.

[49] M. Brezinova, T. Cajka, M. Oseeva, M. Stepan, K. Dadova, L. Rossmeislova, M. Matous, M. Siklova, M. Rossmeisl, O. Kuda, Exercise training induces insulin-sensitizing PAHSAs in adipose tissue of elderly women, Biochim. Biophys. Acta Mol. Cell Biol. Lipids 1865 (2020) 158576.

[50] Q.M. Chen, A.J. Maltagliati, Nrf2 at the heart of oxidative stress and cardiac protection, Physiol. Genom. 50 (2018) 77-97.

[51] S.M. Ahmed, L. Luo, A. Namani, X.J. Wang, X. Tang, Nrf2 signaling pathway: pivotal roles in inflammation, Biochim. Biophys. Acta (BBA) - Mol. Basis Dis. 1863 (2017) 585-597.

[52] S.M. Shoieb, A.A. El-Sherbeni, A.O.S. El-Kadi, Subterminal hydroxyeicosatetraenoic acids: crucial lipid mediators in normal physiology and disease states, Chem. Biol. Interact. 299 (2019) 140-150.

[53] K. Bechynska, N. Daskova, N. Vrzackova, K. Harant, M. Heczkova, K. Podzimkova, M. Bratova, H. Dankova, Z. Berkova, V. Kosek, J. Zelenka, J. Hajslova, R. Sedlacek, J. Suttnar, A. Hlavackova, L. Bartonova, M. Cahova, The effect of omega-3 polyunsaturated fatty acids on the liver lipidome, proteome and bile acid profile: parenteral versus enteral administration, Sci. Rep. 9 (2019) 19097.

[54] J.D. Horton, J.L. Goldstein, M.S. Brown, SREBPs: activators of the complete program of cholesterol and fatty acid synthesis in the liver, J. Clin. Invest. 109 (2002) 1125-1131.

[55] S. Ishii, K. Iizuka, B.C. Miller, K. Uyeda, Carbohydrate response element binding protein directly promotes lipogenic enzyme gene transcription, Proc. Natl.
Acad. Sci. U. S. A. 101 (2004) 15597-15602.

[56] O. Gavrilova, M. Haluzik, K. Matsusue, J.J. Cutson, L. Johnson, K.R. Dietz, C.J. Nicol, C. Vinson, F.J. Gonzalez, M.L. Reitman, Liver peroxisome proliferatoractivated receptor gamma contributes to hepatic steatosis, triglyceride clearance, and regulation of body fat mass, J. Biol. Chem. 278 (2003) 34268-34276.

[57] A. Reis, C.M. Spickett, Chemistry of phospholipid oxidation, Biochim. Biophys. Acta 1818 (2012) 2374-2387.

[58] C.L. Yen, S.J. Stone, S. Koliwad, C. Harris, R.V. Farese Jr., Thematic review series: glycerolipids. DGAT enzymes and triacylglycerol biosynthesis, J. Lipid Res. 49 (2008) 2283-2301.

[59] R. Zechner, R. Zimmermann, T.O. Eichmann, S.D. Kohlwein, G. Haemmerle, A. Lass, F. Madeo, Fat signals - lipases and lipolysis in lipid metabolism and signaling, Cell Metabol. 15 (2012) 279-291.

[60] W.H. Parsons, M.J. Kolar, S.S. Kamat, A.B. Cognetta 3rd, J.J. Hulce, E. Saez, B.B. Kahn, A. Saghatelian, B.F. Cravatt, AIG1 and ADTRP are atypical integral membrane hydrolases that degrade bioactive FAHFAs, Nat. Chem. Biol. 12 (2016) 367-372.

[61] M.J. Kolar, S.S. Kamat, W.H. Parsons, E.A. Homan, T. Maher, O.D. Peroni, I. Syed, K. Fjeld, A. Molven, B.B. Kahn, B.F. Cravatt, A. Saghatelian, Branched fatty acid esters of hydroxy fatty acids are preferred substrates of the MODY8 protein carboxyl ester lipase, Biochemistry 55 (2016) 4636-4641.

[62] M.E. Ertunc, B.P. Kok, W.H. Parsons, J.G. Wang, D. Tan, C.J. Donaldson, A.F.M. Pinto, J.M. Vaughan, N. Ngo, K.M. Lum, C.L. Henry, A.R. Coppola, M.J. Niphakis, B.F. Cravatt, E. Saez, A. Saghatelian, AIG1 and ADTRP are endogenous hydrolases of fatty acid esters of hydroxy fatty acids (FAHFAs) in mice, J. Biol. Chem. 295 (2020) 5891-5905.

[63] D.Y. Hui, P.N. Howles, Carboxyl ester lipase: structure-function relationship and physiological role in lipoprotein metabolism and atherosclerosis, J. Lipid Res. 43 (2002) 2017-2030.

[64] H. Wu, C.M. Ballantyne, Metabolic inflammation and insulin resistance in obesity, Circ. Res. 126 (2020) 1549-1564.

[65] P.V. Dludla, B.B. Nkambule, B. Jack, Z. Mkandla, T. Mutize, S. Silvestri, P. Orlando, L. Tiano, J. Louw, S.E. Mazibuko-Mbeje, Inflammation and oxidative stress in an obese state and the protective effects of gallic acid, Nutrients 11 (2019) 1-29.

[66] M.J. Kolar, S. Konduri, T. Chang, H. Wang, C. McNerlin, L. Ohlsson, M. Harrod, D. Siegel, A. Saghatelian, Linoleic acid esters of hydroxy linoleic acids are antiinflammatory lipids found in plants and mammals, J. Biol. Chem. 294 (27) (2019) 10698-10707.

[67] M. Benlebna, L. Balas, L. Pessemesse, B. Bonafos, G. Fouret, L. Pavlin, B. Goustard, S. Gaillet, T. Durand, C. Coudray, C. Feillet-Coudray, F. Casas, FAHFAs regulate the proliferation of $\mathrm{C} 2 \mathrm{C} 12$ myoblasts and induce a shift toward a more oxidative phenotype in mouse skeletal muscle, Int. J. Mol. Sci. 21 (23) (2020 Nov 28) 9046, https://doi.org/10.3390/ijms21239046.

[68] U. Smith, B.B. Kahn, Adipose tissue regulates insulin sensitivity: role of adipogenesis, de novo li Linoleic acid esters of hydroxy linoleic acids are antiinflammatory lipids found in plants and mammalspogenesis and novel lipids, J. Intern. Med. 280 (2016) 465-475.

[69] P. Zhou, A. Santoro, O.D. Peroni, A.T. Nelson, A. Saghatelian, D. Siegel, B.B. Kahn, PAHSAs enhance hepatic and systemic insulin sensitivity through direct and indirect mechanisms, J. Clin. Invest. 129 (2019) 4138-4150.

[70] I. Syed, M.F. Rubin de Celis, J.F. Mohan, P.M. Moraes-Vieira, A. Vijayakumar, A.T. Nelson, D. Siegel, A. Saghatelian, D. Mathis, B.B. Kahn, PAHSAs attenuate immune responses and promote beta cell survival in autoimmune diabetic mice, J. Clin. Invest. 129 (2019) 3717-3731.

[71] A.R. Schultz Moreira, S. Ruschenbaum, S. Schefczyk, U. Hendgen-Cotta, T. Rassaf, R. Broering, M. Hardtke-Wolenski, L.E. Buitrago-Molina, 9-PAHSA prevents mitochondrial dysfunction and increases the viability of steatotic hepatocytes, Int. J. Mol. Sci. 21 (21) (2020) 8279.

[72] D.S. Im, FFA4 (GPR120) as a fatty acid sensor involved in appetite control, insulin sensitivity and inflammation regulation, Mol. Aspect. Med. 64 (2018) 92-108.

[73] I. Kimura, A. Ichimura, R. Ohue-Kitano, M. Igarashi, Free fatty acid receptors in health and disease, Physiol. Rev. 100 (2020) 171-210.

[74] A. Vella, P. Shah, R. Basu, A. Basu, J.J. Holst, R.A. Rizza, Effect of glucagon-like peptide 1(7-36) amide on glucose effectiveness and insulin action in people with type 2 diabetes, Diabetes 49 (2000) 611-617.

[75] X.H. Wen, Q.L. Guo, J.C. Guo, Effect of 9 - PAHSA on cognitive dysfunction in diabetic mice and its possible mechanism, Biochem. Biophys. Res. Commun. 524 (2020) 525-532.

[76] K. Nishida, Y. Yoshida, M. Itoh, T. Fukada, T. Ohtani, T. Shirogane, T. Atsumi, M. Takahashi-Tezuka, K. Ishihara, M. Hibi, T. Hirano, Gab-family adapter proteins act downstream of cytokine and growth factor receptors and T- and B-cell antigen receptors, Blood 93 (1999) 1809-1816.

[77] Y. Nakaoka, I. Komuro, Gab docking proteins in cardiovascular disease, cancer, and inflammation, Int. J. Inflamm. 2013 (2013) 141068.

[78] J. Lee, P.M. Moraes-Vieira, A. Castoldi, P. Aryal, E.U. Yee, C. Vickers, O. Parnas, C.J. Donaldson, A. Saghatelian, B.B. Kahn, Branched fatty acid esters of hydroxy fatty acids (FAHFAs) protect against colitis by regulating gut innate and adaptive immune responses, J. Biol. Chem. 291 (2016) 22207-22217.

[79] P. Wu, Z. Huang, J. Shan, Z. Luo, N. Zhang, S. Yin, C. Shen, R. Xing, W. Mei, Y. Xiao, B. Xu, J. Mao, P. Wang, Interventional effects of the direct application of "Sanse powder" on knee osteoarthritis in rats as determined from lipidomics via UPLC-Q-Exactive Orbitrap MS, Chin. Med. 15 (2020) 9. 
[80] Y.M. Wang, H.X. Liu, N.Y. Fang, 9-PAHSA promotes browning of white fat via activating G-protein-coupled receptor 120 and inhibiting lipopolysaccharide/ NF-kappa B pathway, Biochem. Biophys. Res. Commun. 506 (2018) 153-160.

[81] P.L. Lakatos, L. Lakatos, Risk for colorectal cancer in ulcerative colitis: changes, causes and management strategies, World J. Gastroenterol. 14 (2008) 3937-3947.

[82] J.P. Rodriguez, C. Guijas, A.M. Astudillo, J.M. Rubio, M.A. Balboa, J. Balsinde, Sequestration of 9-hydroxystearic acid in FAHFA (fatty acid esters of hydroxy fatty acids) as a protective mechanism for colon carcinoma cells to avoid apoptotic cell death, Cancers 11 (4) (2019) 524.

[83] Y.M. Wang, H.X. Liu, N.Y. Fang, High glucose concentration impairs 5-PAHSA activity by inhibiting AMP-activated protein kinase activation and promoting nuclear factor-kappa-B-mediated inflammation, Front. Pharmacol. 9 (2018) 1491.

[84] I. Syed, J. Lee, O.D. Peroni, M.M. Yore, P.M. Moraes-Vieira, A. Santoro, K. Wellenstein, U. Smith, T.E. McGraw, A. Saghatelian, B.B. Kahn,
Methodological issues in studying PAHSA biology: masking PAHSA effects, Cell Metabol. 28 (2018) 543-546.

[85] O. Kuda, On the complexity of PAHSA research, Cell Metabol. 28 (2018) $541-542$.

[86] M.D. Senagolage, M.A. Sommars, K. Ramachandran, C.R. Futtner, Y. Omura, A.L. Allred, J. Wang, C. Yang, D. Procissi, R.M. Evans, X. Han, I.R. Bederman, G.D. Barish, Loss of transcriptional repression by BCL6 confers insulin sensitivity in the setting of obesity, Cell Rep. 25 (2018) 3283-3298.

[87] M.A. Sommars, K. Ramachandran, M.D. Senagolage, C.R. Futtner, D.M. Germain, A.L. Allred, Y. Omura, I.R. Bederman, G.D. Barish, Dynamic repression by BCL6 controls the genome-wide liver response to fasting and steatosis, eLife 8 (2019), e43922.

[88] W.H. Parsons, M.J. Kolar, S.S. Kamat, A.B. Cognetta 3rd, J.J. Hulce, E. Saez B.B. Kahn, A. Saghatelian, B.F. Cravatt, AIG1 and ADTRP are atypical integral membrane hydrolases that degrade bioactive FAHFAs, Nat. Chem. Biol. 12 (2016) 367-372. 